\title{
Dental Implant Considerations in Patients with Periodontal Disease
}

\author{
Radhamoni MadhavanPillai Baiju ${ }^{1 *}$, Biju Thomas ${ }^{2}$ \\ ${ }^{1}$ Department of Periodontics, Government of Dental College, Kottayam, Kerala, India \\ ${ }^{2}$ Professor and Head, Department of Periodontics, AB Shetty Memorial Institute of Dental Sciences, Derlakette, \\ Mangalore, India
}

"Corresponding Author: Radhamoni MadhavanPillai Baiju, Department of. Periodontics, Government of Dental College, Kottayam, Kerala, India; Email: baijurm@gmail.com

Received Date: 21-09-2020; Accepted Date: 29-10-2020; Published Date: 06-11-2020

Copyright $^{\oplus} 2020$ by Baiju RM, et al. All rights reserved. This is an open access article distributed under the terms of the Creative Commons Attribution License, which permits unrestricted use, distribution, and reproduction in any medium, provided the original author and source are credited.

\begin{abstract}
Dental implants have transformed the prosthetic replacement of teeth. Plaque biofilm is the primary etiologic agent for periodontitis and peri-implantitis. Dental plaque biofilm induced inflammation and associated tissue destruction endanger the success and survival of dental implants more than the way it affects natural teeth. Existing periodontitis / history of periodontal disease is considered as a risk factor for peri-implantitis. Meticulous plaque control should be established and comprehensive periodontal therapy executed to obtain periodontal disease stability before dental implant provision. Maintenance of implant is more difficult than maintenance of teeth. Hence extracting teeth for the sake of replacement with implants is not always a good alternative. The general considerations for successful implant placement and maintenance in periodontitis patients are discussed.
\end{abstract}

\section{Keywords}

Peri-implantitis; Dental Implants; Risk Factor; Periodontitis 


\section{Introduction}

Dental implants have transformed the way missing teeth are replaced. Dental plaque biofilm induced inflammation and associated tissue destruction endanger the success and survival of dental implants more than the way it affects natural teeth [1,2]. Periodontitis affects more than $50 \%$ of the population and tooth loss is the hall mark of advanced periodontitis. Comprehensive management of periodontal disease involves not only restoration of periodontal health but also functional and esthetic rehabilitation of the dentition and its long term maintenance. In the last few decades, dental implants have become a suitable option to replace teeth lost due to periodontal disease. Successful treatment of periodontitis and maintenance of periodontal health over the long term is a challenging task. Dental implant supra structure in such patients makes maintenance of periodontal health even more challenging. This paper tries to analyse the evidence regarding dental implant survival in patients with periodontal disease. The general considerations for successful implant placement and maintenance in such patients are also discussed.

\section{Periodontitis as a Risk Factor for Peri-implantitis}

There is enough evidence to consider that existing periodontitis or a history of periodontal disease as a risk factor for peri-implantitis [1]. Presence of residual periodontal pockets more than $5 \mathrm{~mm}$ significantly increase the risk of peri-implant bone loss [2]. Severe periodontitis observed at follow up examination was strongly related to peri-implantitis. In a nine year follow up study among Swedish population, existing periodontal disease reported an odds ratio of 4 for risk of peri-implantitis [3]. According to a systematic review by Heitz Mayfield et al., poor oral hygiene (odds ratio 14) and previous history of periodontal disease (odds ratio 4) are the most important risk factors for periodontal disease [4]. A recent review as part of 2017, classification reported strong evidence linking periodontitis with peri-implantitis [5]. Studies with shorter follow ups failed to report this association, as the impact of implant failure may not be evident within 5 years of implant insertion.

Plaque biofilm is the primary etiologic agent for periodontitis and peri-implantitis. Any kind of periodontal surgery is contra indicated in those who are unable to attain a plaque score less than $20 \%$. It is essential to achieve meticulous self-care (optimum plaque control and control of other risk factors like smoking and diabetes) for the success of periodontal therapy. Deep periodontal pockets that harbor microorganisms pose threat to the health of an implant placed adjacent to it. Therefore it is advisable to follow the guidelines of periodontal therapy, treat all existing periodontal disease and address all plaque retentive factors before embarking upon dental implant insertion. Periodontal stability has to be achieved (pocket depths less than or equal to $3 \mathrm{~mm}$ with bleeding score less than $10 \%$ ) prior to dental implant placement. 


\section{Maintenance of Periodontally Compromised Teeth Vs Replacement with Implants}

Whether to extract teeth with poor periodontal prognosis and replace with dental implants has always been a clinical dilemma. Classical papers on long term follow up (more than 20 years) of successfully treated and well maintained periodontally compromised dentition with advanced bone loss and furcation involvements demonstrated good survival rates for teeth $[5,6]$. It shows that presence of advanced bone loss or furcation involvement is not an indication for extraction and replacement. Available evidence suggests that periodontally compromised teeth could be as successful as implants in the long term if maintained properly. With simple non-surgical therapy and systematic regular supportive care, teeth with poor support can function adequately and free of symptoms for many years. Hence, considering the fact that maintenance of implant is more difficult than maintenance of teeth, extracting teeth for the sake of replacement with implants is not always a good alternative. Implants have a finite life time and should be considered as late as possible, unless it is advised otherwise for obvious clinical reasons.

\section{Clinical Considerations}

Number of Implants and Implant Position: Implant planning including the number of implants, their location and prosthesis design should start before extraction. A diagnostic wax up and surgical stent will help plan and execute surgery. The ideal implant position should ensure adequate bone and soft tissue coverage, esthetic emergence and a cleansable superstructure. The concept of over engineering (too many implants to prevent occlusal over loading) is not advisable. Implant fixture should be distanced $3 \mathrm{~mm}$ from each other and 1.5 $\mathrm{mm}$ from natural tooth. Vertically the implant shoulder should be within $5 \mathrm{~mm}$ from the proximal contact and $3 \mathrm{~mm}$ from the buccal gingival margin of adjacent teeth. Implants of inappropriate diameter that are placed too deep or too buccally or palatally will result in accelerated bone loss and soft tissue destruction.

Supra Structure Design: Implant abutment margins may be located sub gingivally in the aesthetic zone but this may lead to suboptimal marginal fit attracting plaque. Where ever possible margins should be placed supra gingivally especially with, multiple abutments.

Screw Vs Cement: Screw retained restorations are preferred as it is difficult to remove excess cement especially in case of subgingival margins or with complicated multi-unit supra structure. The use of retraction cord during cementation and floss post-cementation are encouraged to remove cement excess. A radiograph taken immediately after cementation helps to detect excess cement a serves as a base line for surveillance of peri-implant bone loss. There 
have to be minimum of $5 \mathrm{~mm}$ restorative space to employ screws and occasional screw loosening have to be considered. A loose screw may help track biofilm deeper and may lead to peri implant mucositis or even peri-implantitis.

Oral Hygiene Recommendations: Meticulous oral hygiene maintenance as part of the periodontal maintenance protocol, with extra efforts extended to implants is vital in treated periorontitis patients. Daily flossing for single tooth implants with full papillae and optimal contacts is recommended, Floss offer little help in treated periodontitis patients. Rightly fitting interdental brushes are preferred for reduced periodontium around implants. Areas under the pontics and around the abutments should be cleaned. In case of multiple implant restorations super floss may be employed.

Maintenance Care Schedule: The fact that "once a periodontitis patient, always a periodontitis patient", points to the importance of strict adherence to an individually tailored implant maintenance regimen to all periodontitis patients post treatment. Among this group those with systemic risk factors like diabetes or smoking and those with bulky supra structures or less than ideal implant positions need to be more frequently reviewed.

Removable Vs Fixed: Substantial bone and soft tissue loss associated with long edentulous sites in periodontitis patients may be better managed by implant supported removable prosthesis like over dentures. Removable supra structures make self-care better and may offer better esthetic replacement of pink structures.

\section{Discussion}

Replacement of missing teeth in a periodontally compromised patient is a challenging task that has to be meticulously planned and discussed with the patient. It should be considered that it is always better to treat and maintain a periodontally compromised tooth that is functional and asymptomatic [7,8]. Several papers that reported long term follow up of teeth with advanced bone loss and extensive furcation involvement and those treated with respective therapy have concluded that periodontally compromised teeth could be as successfully maintained in the long term as dental implants [9]. Dental implant restorations too have a finite life and hence cannot be considered as an alternative to teeth, irrespective of its poor long term prognosis. So provision of dental implants in a periodontitis patient warrants careful consideration of various factors described above that could influence the long term success and survival of Osseo integrated implants.

Existing periodontitis is now regarded as a risk factor for peri-implantitis with an odds ratio of 4. History of periodontal disease increases the risk of future biologic complications even in a well-treated and maintained periodontal patient. Presence of chronic periodontitis increases the 
risk of peri-implantitis by 3 times whereas aggressive periodontitis increases the risk up to 13 times [1]. Presence of periodontitis detected at implant follow ups is a strong indicator of periimplantitis [10].

The importance of individually tailored maintenance protocol based on potential risk factors cannot be over emphasized in imparting long term success of implant supported removable and fixed restorations. A radiograph should be taken soon after placement of the prosthesis and pocket charts recorded as this serves as baseline data for monitoring peri implant bone level and soft tissue health [11]. There is strong evidence from various papers that poor adherence to or lack of adequate maintenance care leads to peri-implantitis [12-14]. The width and thickness of keratinised gingiva around implants is another critical aspect that determine the success of implant [15].

Karoussis et al., reported that dental implants in patients with a history of periodontitis had deeper pockets and greater peri-implant bone loss suggestive of peri-implantitis [16]. Treated periodontitis patients requiring implant supported restorations often need to undergo ridge augmentation procedures in the prospective implant sites due to ensuing alveolar bone loss. But a systematic review of controlled clinical trials could not find any difference in outcomes among implants inserted in augmented bone compared to native bone [17]. Meticulous assessment of the implant site, proper planning of the position and number of implants, well designed prosthesis and individually tailored implant maintenance are the crucial factors for the success of implant therapy in periodontitis patients.

\section{Conclusion}

Periodontitis could be the result of inability to maintain adequate plaque control in a susceptible patient. Such patients may find it hard to maintain complex and more critical implant crowns or bridges. The clinical judgment has to be wisely done before planning to replace teeth with reduced support with implants in such patients. The importance of risk assessment, considering all potential factors that could predispose inflammation and bone destruction around as osseo integrated implant is highlighted.

\section{References}

1. Sgolastra F, Petrucci A, Severino M, Gatto R, Monaco A. Periodontitis, implant loss and peri implantitis. A meta analysis. Clin Oral Implants Res. 2015;26(4):e8-16.

2. Pjetursson BE, Helbling C, Weber HP, Matuliene G, Salvi GE, Brägger U et al. Peri implantitis susceptibility as it relates to periodontal therapy and supportive care. Clin Oral Implants Res. 2012;23(7):888-94.

3. Derks J, Schaller D, Hakansson J, Wennstrom JL, Tomaci C, Berglundh T. Effectiveness of implant therapy analyzed in a Swedish population: prevalence of peri implantitis. J Dent Res. 2016;95(1):43-9.

4. Heitz-Mayfield LJ,Mombelli A. The therapy of periimplantitis: a systematic review. Int J Oral Maxillofac Implants. 2014;29:325-45. 
5. Schwarz F, Derks J, Manje A, Wang HL. Peri implantitis. J Periodontol. 2018;89:S267-90.

6. Axelsson P, Nystrom B, Lindhe J. The long term effect of a plaque control programme on tooth mortality, caries and periodontal disease in adults. Results after 30 years of maintenance. J Clin Periodontol. 2004;31(9):749-57.

7. Hirschfeld L, Wasserman B. A long term survey of tooth loss in 600 treated periodontal patients. J Periodontol. 1978;49(5):225-37.

8. Patel RM. Dental Implants for patients with periodontitis. Prim Dent J. 2019;8(4):54-61.

9. Fugazzotto PA. A comparison of the success of root resected molars and molar position implants in function in a private practice: results of up to 15 plus years. J Periodontol. 2001;72(8):1113-23.

10. Doubert DM, Weinstein BF, Bordin S, Leroux BG, Flemmig TF. Prevalence and predictive factors for peri implant disease and implant failure: a cross sectional analysis. J Periodontol. 2015;86(3):337-47.

11. Alani A, Kelleher M, Bishop K. Peri implantitis, Part 1: Scope of the problem. British Dent J. 2014;217(6):281-7.

12. Romanos Ge, Delgado-Ruiz R, Sculean A. Concepts for prevention of implant therapy. Periodontol 2000. 2019;81:7-17.

13. Rocuzzo M, Bonino F, Aglietta M, dalmasso P. Ten year results of a three arms prospective cohort study on implants in periodontally compromised patients. Part 2: clinical results. Clin Oral Implants Res. 2012;23(4):389-95.

14. Cortellini S, Favril C, De Nutte M,Teughels W, Quirynen M. Patient compliance as a risk factor for the outcome of implant treatment. In: Romanos GE et al. Risks and their prevention in implant therapy. Periodontal 2000. 2019;81(1):209-25.

15. Thoma DS, Naenni N, Figuero E, Hämmerle CHF, Schwarz F, Jung RE, et al. Effects of soft tissue augmentation procedures on peri-implant health or disease: A systematic review and meta-analysis. Clin Oral Implants Res. 2018;29(15):32-49.

16. Karoussis IK, Kotsovilis S, Fourmousis I. A comprehensive and critical review of dental implant prognosis in periodontally compromised partially edentulous patients. Clin Oral Implants Res. 2007;18(6):669-79.

17. Ha"mmerle CH, Jung RE, Feloutzis A. A systematic review of the survival of implants in bone sites augmented with barrier membranes (guided bone regeneration) in partially edentulous patients. J Clin Periodontol. 2002;29(Suppl 3):226-33. 